\title{
Degradation Mechanisms in Organic Lead Halide Perovskite Light-Emitting Diodes
}

Vittal Prakasam, Daniel Tordera, Henk J. Bolink, Gerwin Gelinck*

Vittal Prakasam, Dr. Daniel Tordera, Prof. Gerwin Gelinck

Holst Centre, High Tech Campus 31, 5656 AE, Eindhoven, The Netherlands

E-mail: gerwin.gelinck@tno.nl

Vittal Prakasam, Prof. Henk J. Bolink

Instituto de Ciencia Molecular, Universidad de Valencia, C/Catedrático J. Beltrán 2, 46980

Paterna, Spain

Prof. Gerwin Gelinck

Department of Applied Physics, Eindhoven University of Technology, 5600 MB Eindhoven, The Netherlands

Keywords: metal halide perovskites, light emitting diodes, degradation, device lifetime, perovskite stability

\begin{abstract}
Organic-inorganic metal halide perovskites have attracted significant attention for lowcost, high-efficiency, color-pure light-emitting applications. However, as seen in many reports so-far, perovskite light-emitting diodes (PeLED) suffer from poor operational lifetime, limiting their practical use. The underlying degradation mechanism is a topic of crucial importance. Here, the degradation mechanisms of methylammonium lead bromide based PeLED are investigated. When the PeLED is electrically biased, there is an initial raise in the luminance followed by a rapid reduction in luminance and current density. Microscopic studies reveal the formation of $\mu \mathrm{m}$ sized spots that are photoluminescent but not electroluminescent. It is demonstrated that this degradation is due to the formation of gaseous compounds that leads to local delamination of the cathode, thereby reducing the electroluminescence. When the degraded cathode is substituted with a fresh cathode, the initial luminance is largely recovered. By further analyzing the buried interface of the cathode, the formation of lead bromide and gaseous methylamine due to the degradation of the perovskite layer are revealed. These insights
\end{abstract}




\section{WILEY-VCH}

will help to further improve the lifetime of PeLEDs. As an example, it is shown that substituting methylammonium cations by cesium leads to longer lifetimes.

\section{Main Text}

Organic-inorganic metal halide perovskite (perovskite) materials have attracted significant attention for light emitting applications such as light-emitting diodes ${ }^{[1-3]}$ (LEDs) and lasing ${ }^{[4,5]}$ driven by their favorable properties such as tunable bandgap, ${ }^{[6]}$ high emission color purity $^{[7]}$ and high charge carrier mobilities ${ }^{[8]}$ in addition to its low material cost. ${ }^{[9]}$ Several strategies have been developed to improve the radiative recombination rate in the perovskite layer such as compositional tuning, ${ }^{[10-12]}$ synthesis of low dimensional perovskite with bulky cations $^{[13,14]}$ and use of ultra-thin emitting layers. ${ }^{[15]}$ These strategies combined with passivation techniques have led to the demonstration of perovskite light emitting diodes (PeLEDs) with external quantum efficiencies (EQE) above $20 \%^{[12]}$ in the visible and $21.6 \%$ in the near infrared regions, just short of the best organic and quantum dot LEDs. ${ }^{[3]}$ These attributes make them one of the most promising candidate for next-generation low-cost high efficiency light-emitting materials.

However, one of the foremost impediments for PeLEDs is their relatively poor operational lifetime. The typical operational lifetimes ( $\mathrm{LT}_{50}$, time taken to reach half the initial luminance) of PeLEDs can range between a few minutes to hours ${ }^{[3,12,16]}$ depending on the experimental conditions (perovskite composition, bias, encapsulation). Recent electrical studies suggest that reduction of trap states under short-lived electrical bias (70 $\mu \mathrm{s})$ improves the device

performance and postulate creation of detrimental halide vacancies under continuous bias. ${ }^{[17,18]}$ Similar enhancement has also been observed under low background bias $(<3 \mathrm{~V})$ conditions. ${ }^{[19]}$ Despite these developments, there is a lack of thorough understanding of the origin of PeLED degradation under continuous electrical bias, particularly at current densities and brightness levels relevant for LED applications. 


\section{WILEY-VCH}

Here, we elucidate the degradation mechanisms of methylammonium lead tribromide $\left(\mathrm{MAPbBr}_{3}\right)$ perovskite based LEDs under continuous bias. We show that after an initial rise in luminance, both the current density and luminance drop by several orders of magnitude within a few minutes ( $\sim$ mins). Microscopic images reveal the formation and growth of $\mu$ m-sized circular spots that exhibit photoluminescence (PL) characteristic of $\mathrm{MAPbBr}_{3}$ but no electroluminescence (EL). Remarkably, we show that the overall luminance and current density of seemingly degraded PeLEDs can be largely recovered by peeling off and depositing a fresh cathode, indicating that the reduction in device performance is due to cathode impairment. By investigating the degraded PeLEDs using scanning electron microscopy (SEM), structural characterization techniques and energy-dispersive X-ray (EDX) profiling we propose that in PeLEDs the perovskite material dissociates under an electric bias producing gaseous compounds. The concomitant pressure build-up causes the cathode to delaminate locally from the perovskite film. This work brings crucial insights into the degradation mechanisms currently challenging PeLEDs and possible mitigation routes.

Figure 1a shows the PeLED stack used in this work. The device consists of a $130 \mathrm{~nm}$ indium tin oxide (ITO) anode, a $100 \mathrm{~nm}$ poly(3,4-ethylenedioxythiophene)poly(styrenesulfonate) (PEDOT:PSS) hole injection layer, a $30 \mathrm{~nm}$ N4,N40-(biphenyl-4,40diyl)bis(N40-(naphthalen-1-yl)-N4,N40-diphenylbi-phenyl-4,40-diamine) (Di-NPB) hole transport layer, $80 \mathrm{~nm}$ of $\mathrm{MAPbBr}_{3}$ (in 2:1 ratio) as the emitting perovskite, a $30 \mathrm{~nm}$ 1,3-bis[3,5di-(pyridin-3-yl)phenyl]benzene (BmPyPhB) electron transport layer (ETL) and a $\mathrm{LiF} / \mathrm{Al}$ $(1 / 100 \mathrm{~nm})$ cathode, that were optimized in an earlier work. ${ }^{[11]}$ The energy levels of the different device layers are illustrated in Figure 1b, which shows that the transport layers are well matched to contain the injected charges within the perovskite layer. The current density-voltageluminance $(J-V-L)$ curves of the PeLED are shown in Figure 1c. The PeLED has a low leakage current of $\sim 10^{-5} \mathrm{~mA} / \mathrm{cm}^{2}$, a turn-on voltage of $3 \mathrm{~V}$ and reaches a maximum luminance of $\sim 17800$ $\mathrm{cd} / \mathrm{m}^{2}$. This corresponds to a current efficiency of $14 \mathrm{~cd} / \mathrm{A}$ and an EQE of $3.9 \%$. The 


\section{WILEY-VCH}

electroluminescence spectrum (inset Figure 1c) shows that it emits in the green region, with a peak centered around $\sim 530 \mathrm{~nm}$.

Firstly, it is important to exclude any environmental degradation effects on the $\mathrm{MAPbBr} 3$ based PeLEDs. Previous studies have shown the degradation of wide band-gap iodide based perovskite in the presence of moisture through water catalysis. ${ }^{[20]}$ It was also postulated that the addition of bromide into the perovskite lattice might improve their resistance towards water catalysis. ${ }^{[21]}$ Therefore, to probe the effects of the ambient conditions such as the presence of oxygen and moisture, we fabricated PeLEDs by spin-coating and annealing the $\mathrm{MAPbBr} 3$ layer either in the presence of compressed dry air $\left(20 \% \mathrm{O}_{2},<1 \%\right.$ RH-relative humidity) or humid air (20\% $\left.\mathrm{O}_{2}, \sim 55 \% \mathrm{RH}\right)$. The $J$ - $V$ - $L$ curves of these PeLEDs (Figure $1 \mathrm{~d}$ ) show that the mere presence of $\mathrm{O}_{2}$ has no effect on the device performance; devices fabricated in $\mathrm{N}_{2}$ (black line) and $\mathrm{O}_{2}$ (red line) ambients show similar current densities and luminances (shown here for an average of two devices). However, in the presence of humid air (cyan line) the device performance is significantly compromised, showing a reduction in both current density and luminance besides non-uniform emission (inset Figure 1d).

PeLEDs were also found to degrade upon storage over a long period of time (3 days) in a $\mathrm{N}_{2}$ filled glove box. This is most likely due to the inevitable ingress of water molecules (moisture level in the glovebox was ca. 5 ppm), as PeLEDs that were encapsulated using a highquality thin-film moisture barrier with a very low water vapor transmission rate of $<10^{-6}$ $\mathrm{g} / \mathrm{m}^{2} /$ day $^{[22]}$ do not show significant degradation during storage (Figure S1). Therefore, for further tests, we encapsulated the PeLEDs with a thin-film moisture barrier immediately after the cathode deposition (refer to Figure S2 for details).

Figure 2a shows the current density and luminance of the PeLEDs under continuous bias as a function of time. The range of voltages $(4 \mathrm{~V}, 5 \mathrm{~V}$ and $6 \mathrm{~V})$ was chosen to reflect the luminance levels relevant for display $\left(300-1000 \mathrm{~cd} / \mathrm{m}^{2}\right)$ and signage applications (>2000 $\mathrm{cd} / \mathrm{m}^{2}$ ), and since higher bias voltages ( $>6 \mathrm{~V}$ ) lead to catastrophic failure of the devices. The 


\section{WILEY-VCH}

current density decreases by $\sim 40 \%$ in the first $10 \mathrm{~s}$, followed by an accelerated decrease with time for all three biasing conditions. However, the luminance increases by ca. $\sim 80 \%$ reaching peak intensities at $4 \mathrm{~s}, 7 \mathrm{~s}$ and $10 \mathrm{~s}$ at $6 \mathrm{~V}, 5 \mathrm{~V}$ and $4 \mathrm{~V}$ bias respectively. This is then followed by a dramatic drop in luminance to ca. $<10 \%$ of the initial value after 5 min biasing. This corresponds to a peak luminance $\mathrm{LT}_{50}$ of $46 \mathrm{~s}, 22 \mathrm{~s}$ and $12 \mathrm{~s}$ at $4 \mathrm{~V}, 5 \mathrm{~V}$ and $6 \mathrm{~V}$, respectively. The initial rise in luminance may be attributed to filling of non-radiative recombination trap sites by excess mobile ions, as reported earlier. ${ }^{[17,19]}$ Steady-state PL measurements also show a rise in luminescence with time (Figure S3) which further corroborates the trap filling mechanism. ${ }^{[23]}$ While the PL changes were reversible (Figure S3), even after prolonged excitation (30 min), the electroluminescence (EL) changes were irreversible (Figure S4).

Next, in-situ imaging techniques were used to probe the degradation mechanisms. PeLEDs were biased in-situ on a microscope under bright field (BF), fluorescent (405 nm excitation) and recording modes, to capture the morphological, PL and EL evolution, respectively. Figure 2b compares the BF, PL and EL microscope images of a pristine PeLED with that of a PeLED that was biased for 5 minutes at $5 \mathrm{~V}$. The pristine PeLED shows a smooth texture and uniform green PL and EL emission, characteristic of $\mathrm{MAPbBr}_{3}$, except for some local defects due to particles (indicated with white arrows in Figure 2b). After 1-2 minutes, small dark spots appear (Figure S5a) in the EL image, that grow in size over time. The radial growth of the spots is not continuous but intermittent over time (see video in SI at 10x speed). After biasing for 5 minutes, circular spots of $\sim 70 \mu$ m (measured over an area of $500 \times 500 \mu \mathrm{m}^{2}$ ) are observed. In parallel, the EL reduces with time over the complete device area. BF images also reveal the formation of the circular features. Interestingly, the formed circular spots with no EL exhibit PL characteristic of $\mathrm{MAPbBr}_{3}$. In fact, the PL in the circular spots seems a little higher than its surroundings after extended bias (Figure S5b). This observation of the formation of spots that show PL but no EL can be explained by local delamination of one layer from another in the multi-layered PeLEDs. Such delamination would restrict the current injection 


\section{WILEY-VCH}

locally, explaining the loss in EL but would not a priori affect the photoluminescence of the perovskite film. The delamination is expected to occur at the weakest interface between the adjacent layers in the PeLED. To verify this, we manually stripped off the top encapsulation foil (PET with the encapsulation layers) from the glass substrate containing the (degraded) PeLEDs. The cathode layer ( $\mathrm{LiF} / \mathrm{Al}$ ) readily peels off along with the encapsulation foil. The ETL and the other PeLED layers remain on the glass substrate. This stripping technique allows us to study the buried interfaces. It also allows us to deposit a new $\mathrm{LiF} / \mathrm{Al}$ cathode onto a degraded device and validate the cathode delamination prognosis. Figures 2c,d show the $J$ - $V$ - $L$ curves of a PeLED in pristine state (black lines), post $5 \mathrm{~V}$ bias for 5 min (blue lines) and the characteristics of the same device with re-deposited cathode (red lines). The current density and luminance fall to less than $5 \%$ of their initial intensities after biasing. Remarkably, after stripping and re-depositing the cathode, the luminance is restored to intensities similar to the pristine device and with the same uniform spectral emission (inset Figure 2d). The leakage current increases from $10^{-6} \mathrm{~mA} / \mathrm{cm}^{2}$ to $\sim 10^{-2} \mathrm{~mA} / \mathrm{cm}^{2}$ suggesting possible creation of pinholes during the peel off procedure. Crucially, the luminance recovery strongly suggests that the cathode is indeed impaired when the PeLED is biased, impeding charge injection locally and explains the dark spots in EL images (refer Figure S6 and Table T1 for a comparison of device metrics).

To investigate possible degradation of the perovskite film, cross-sectional SEM images of the PeLED before and after biasing ( $5 \mathrm{~V}$ for $5 \mathrm{~min}$ ) were captured. The pristine device shows a continuous, dense polycrystalline perovskite film while the biased device reveals the formation of cavities (indicated by the yellow circles) within the bulk of the perovskite layer, as shown in Figures 3a and S7. The formed cavities seem to be concentrated at the Di-NPB/ $\mathrm{MAPbBr}_{3}$ interface. These results allow us to postulate the following mechanism. Part of the $\mathrm{MAPbBr} 3$ perovskite degrades under the influence of an electric field/current and forms gaseous 


\section{WILEY-VCH}

degradation compounds. These gaseous compounds build-up pressure inside the device and eventually delaminate the $\mathrm{LiF} / \mathrm{Al}$ layer which restricts current injection.

Next, the SEM images of the ETL of a biased PeLED after stripping off the cathode were captured using secondary electron (SE) and variable pressure secondary electron (VPSE) detectors as shown in Figure 3b. The SE image clearly shows circular patterns similar to that observed in BF microscopic images in degraded devices, confirming that the observed patterns are indeed related to changes at the cathode side. The mild roughness visible in the image is due to miniscule difference in film thickness due to the peeling. These features are absent in the circular areas, which further suggests delamination between these areas and the cathode. Figure $3 \mathrm{~b}$ shows the image from the VPSE detector which can record panchromatic cathodoluminescence of the sample, i.e., the PL of the sample due to secondary electron bombardment from $\sim 1 \mu \mathrm{m}$ depth. ${ }^{[24]}$ Similar to the fluorescent microscopic images, the VPSE image shows that the circular areas have higher photoluminescence intensities (from the perovskite) than the rest of the film. This can be explained as the consequence of cathode delamination. The local delamination of the cathode during device operation effectively impede current injection into the perovskite layer and this stops the electric field induced degradation of the perovskite layer itself. Moreover, the circular patterns also have scratches/holes within them, an indication of an out-gassing pathway from the perovskite layer through the ETL. Figure 3c shows the X-ray diffraction (XRD) patterns collected from complete PeLEDs, in pristine state and after biasing at $5 \mathrm{~V}$ for various duration (5, 10 \& $15 \mathrm{~min})$. The XRD peaks at $15.0^{\circ}, 30.0^{\circ}, 33.6^{\circ}$ and $35.6^{\circ}$ are characteristic of the cubic phase of $\mathrm{MAPbBr}_{3},{ }^{[25]}$ and the peak at $38.4^{\circ}$ can be ascribed to the (111) plane of $\mathrm{Al}$ cathode. The kink in the background is due to the reflections from the encapsulation layer (Figure S9). Clearly, there is a drop in the $15.0^{\circ}$ and $30.0^{\circ}$ perovskite peaks with an increase in bias timings from 0 to $15 \mathrm{~min}$, further confirming the degradation of $\mathrm{MAPbBr}_{3}$ in the stack. The XRD patterns collected from biased PeLEDs 


\section{WILEY-VCH}

after stripping off the cathode under inert atmosphere shows the presence of additional peaks that can be attributed to $\mathrm{PbBr}_{2}$ (Figure S10).

We also imaged the stripped off cathode on the encapsulation foil using SEM to investigate the degradation compounds, as shown in Figure 4a. The pristine cathode (4a 1.), stripped off a pristine PeLED, is composed of pure $\mathrm{Al}$ and appears smooth, except for the wrinkles from the encapsulation foil behind. The cathode stripped off a biased PeLED (5V for 5 min.) (4a 2., 3.) shows the presence of multiple circular features. By varying the focal length of the SEM image, we found that the circular features are local recessions, i.e., valleys. Higher magnification image reveals concentric rings inside the valleys (4a 3.). These rings are likely associated with the intermittent growth of the spot, as evidenced in the microscopic images of the biased devices. The SEM image in Figure $4 \mathrm{~b}$ was taken from the central region within a valley. Using EDX analysis we found that the darker regions in Figure 4b are composed of pure $\mathrm{Al}$. The lighter regions show the presence of both $\mathrm{Al}$ and $\mathrm{Pb}$ in the EDX spectra ( $\mathrm{C}$ and $\mathrm{O}$ signal come from the foil). Similar studies on a PeLED with Au cathode instead of LiF/Al, shows analogous features, and the presence of bromide in sections within the valleys (see Figure S11). Therefore, we assume that the lighter regions inside the valleys in the Al cathode also have bromide in them but are undetectable due to the overlapping EDX spectra of $\mathrm{Al}$ and $\mathrm{Br}$. Based on these results, we propose that under electric bias the organic part of the $\mathrm{MAPbBr}_{3}$ readily decomposes into methylamine $\left(\mathrm{CH}_{3} \mathrm{NH}_{2}\right)$ and lead(II)bromide $\left(\mathrm{PbBr}_{2}\right)$, and the remains of which $\left(\mathrm{H}^{+}\right.$and $\left.\mathrm{Br}^{-}\right)$can form hydrogen bromide, ${ }^{[26]}$ as per the Equation 1.

$$
\left[\mathrm{CH}_{3} \mathrm{NH}_{3} \mathrm{PbBr}_{3}\right]_{\mathrm{n}} \rightarrow\left[\mathrm{CH}_{3} \mathrm{NH}_{3} \mathrm{PbBr}_{3}\right]_{\mathrm{n}-1}+\mathrm{CH}_{3} \mathrm{NH}_{2}(\mathrm{~g})+\mathrm{PbBr}_{2}+\mathrm{HBr}(\mathrm{g})
$$

Both methylamine and hydrogen bromide have a low boiling of $-66^{\circ} \mathrm{C}$ and $-6{ }^{\circ} \mathrm{C}$, and thus will exist as gases at room temperature. ${ }^{[27,28]}$ Indirect evidence for the proposed decomposition of $\mathrm{MAPbBr} r_{3}$ comes from the fact that such degradation features are not observed in $\mathrm{CsPBr}_{3}$ PeLED as shown in Figure S12. 


\section{WILEY-VCH}

Finally, we rule out the impact of Joule heating as the dominant degradation mechanism. We recorded the time evolution of the temperature of the PeLEDs while biasing, using a high-precision thermocouple probe attached to the cathode of the PeLEDs. The results (Figure S13a) show that the temperatures gradually rise and reach a maximum of $28{ }^{\circ} \mathrm{C}, 35^{\circ} \mathrm{C}$ and $48{ }^{\circ} \mathrm{C}$ after $\sim 15 \mathrm{~s}$ into the biasing at $4 \mathrm{~V}, 5 \mathrm{~V}$ and $6 \mathrm{~V}$ bias respectively, before dropping back to room temperature. To further confirm this, we captured infrared thermography images of the PeLEDs through the glass side, where similar temperatures were recorded $\left(30{ }^{\circ} \mathrm{C}, 38{ }^{\circ} \mathrm{C}\right.$ and 58 ${ }^{\circ} \mathrm{C}$ after $15 \mathrm{~s}$ for $4 \mathrm{~V}, 5 \mathrm{~V}$ and $6 \mathrm{~V}$ respectively) within the device (Figure S13b). Previously, Joule heating has been suggested as a crucial driving factor in PeLED degradation. ${ }^{[14,15,19]}$ Thermal decomposition of $\mathrm{MAPbBr} 3$ perovskite has been observed starting at $130{ }^{\circ} \mathrm{C} .{ }^{[29]}$ Annealing the PeLEDs to above $180{ }^{\circ} \mathrm{C}$ does result in the creation of visible degradation features (Figure S14). However, based on the surface temperature estimates, it is unlikely that the perovskite layer will get sufficiently hot to induce detrimental thermal degradation under bias. Thus, we postulate that Joule heating is unlikely to be the preeminent cause of degradation in PeLEDs containing organic cations but will likely contribute a little to the overall degradation process.

In summary, we studied the degradation of $\mathrm{MAPbBr}_{3} \mathrm{PeLED}$ under operational conditions. The observed decrease in current density and luminance is mainly driven by the decomposition of the $\mathrm{MAPbBr}_{3}$, leading to the formation of methylamine, lead(II) bromide and hydrogen bromide. The formation of the gaseous methylamine and hydrogen bromide increases the pressure inside the device and leads to local delamination of the cathode off the ETL. This explains the appearance of dark spots in the EL image of biased PeLED. The dark spots growth eventually stalls due to the decrease in the bias current as a consequence of cathode delamination. The formation of $\mathrm{PbBr}_{2}$ would further contribute to the quenching of luminance and deterioration of the cathode by virtue of ion migration and redox reactions as reported earlier. ${ }^{[31,32]}$ Remarkably, the luminance and current densities can be largely restored in the PeLEDs by stripping off and re-depositing a new cathode. The degradation occurs only in the 


\section{WILEY-VCH}

presence of electric field/charges, an effect similar to that reported earlier for methylammonium lead iodide based perovskite. ${ }^{[30]}$ Meanwhile, Joule heating has a negligible effect but is likely to accelerate the degradation process at the current densities relevant for LED application.

While great strides of improvement have been demonstrated in the recent years in terms of device efficiencies and spectral response, substantial progress must still be made to improve the operational lifetime of PeLEDs. Any perovskite with methylammonium cation is expected to degrade in a similar fashion as reported here, at least in part, under forward bias. In order to improve the operational lifetime of PeLEDs, further research should focus on using perovskite materials with more stable cations such as $\mathrm{CsPbBr}$. In this respect, it is noteworthy that recently high $\mathrm{LT}_{50}$ of $250 \mathrm{~h}^{[33]}$ was reported for $\mathrm{CsPbBr}_{3}$ based PeLEDs.

\section{Experimental Section}

PeLED fabrication: Glass substrates with pre-patterned indium tin oxide (ITO), purchased from Naranjo Substrates B.V., were washed with soap (1\% Teepol) and rinsed with deionized water in an ultrasonic bath and then in a megasonic bath. The substrates were then subjected to $\mathrm{O}_{2}$ plasma for 3 minutes. Then, PEDOT:PSS (Clevios P AI4083) was filtered through a 0.45 $\mu \mathrm{m}$ PVDF (polyvinylidene difluoride) filter and spin-coated on the substrates, followed by 15 min annealing at $130^{\circ} \mathrm{C}$. Di-NPB (Lumtec Corp.) in toluene (0.6 wt/v\%) was spin-coated on top of the PEDOT:PSS layer in a glovebox, followed by 10 min annealing at $110{ }^{\circ} \mathrm{C}$. The $\mathrm{Di}$ NPB layer was then treated with $\mathrm{N}_{2}$ plasma for $30 \mathrm{~s}$ at $1000 \mathrm{~W}$ to improve surface wetting. The perovskite solution was prepared by dissolving methylammonium bromide (GreatCell Solar) or cesium bromide (GreatCell Solar) and Lead(II)bromide (TCI Chemicals) in DMSO (0.8 M for $\mathrm{MAPbBr}_{3}$ or $0.75 \mathrm{M}$ for $\mathrm{CsPbBr}_{3}$ ) in 2:1 ratio, respectively. The solutions were stirred with a magnetic stirrer at $50^{\circ} \mathrm{C}$ overnight before use. The perovskite layer was spin-coated on top of the Di-NPB layer. Whilst spinning the perovskite layer, $145 \mu \mathrm{l}$ of chlorobenzene was dripped on top of the wet layer to induce perovskite crystallization. The coated substrates were immediately annealed at $80^{\circ} \mathrm{C}$ for 2 minutes. Despite the use of an excess MABr in the precursor 
solution, the final $\mathrm{MAPbBr}_{3}$ films did not contain any excess crystalline $\mathrm{MABr} .{ }^{[11]}$ For the ambient condition experiments, the glove box was filled with dry air or humid air before processing the perovskite layer. The spin settings were tuned to result in desired layer thicknesses as indicated in the main text. The substrates were then transferred to a thermal evaporator (Lesker) where the desired thickness of BmPyPhB (Lumtec Corp.), lithium fluoride and aluminum layers were consecutively deposited through a mask, at a vacuum pressure of < $1 \times 10^{-6}$ mbar.

Encapsulation layer fabrication and Cathode Peeling: A PET (Polyethylene terephthalate) foil was coated with an organic layer for planarization (OCP), followed by the deposition of SiN layer using plasma enhanced chemical vapor deposition technique ${ }^{[22]}$. A commercial pressure sensitive adhesive (PSA) layer is then laminated on top of the SiN layer. The foil is then cut into desired shapes and annealed at $90{ }^{\circ} \mathrm{C}$ for one hour in a vacuum oven before using for encapsulation. The encapsulated PeLEDs were first heated at $110{ }^{\circ} \mathrm{C}$ for 1 minute to delaminate the adhesive from the PET foil and bind to the Al cathode. Then the samples were cooled down to room temperature, which transfers the Al cathode to the adhesive and binds to the PET foil. The foil was then carefully stripped from the corners using a knife.

Characterization: The current density-voltage-luminance characteristics were measured in a glovebox using a Keithley 2400 source meter and a calibrated silicon photodiode (against a Minolta LS-110 luminance meter) connected to a Keithley 6485 Picoammeter, at a step rate of $200 \mathrm{mV}$. The electroluminescence spectra were obtained using an Oceanoptics USB 2000+ spectrophotometer. The in-situ microscopic images were captured by biasing the encapsulated samples underneath a Leica INM 100 microscope (MC120HD camera). The photoluminescent images were captured by exciting the samples using a mercury arc lamp cut off with a bandpass filter at $405 \mathrm{~nm}$. The topographical SEM images were measured with a Zeiss Supra 55VP machine using the secondary electron or the variable pressure secondary electron detector. An 


\section{WILEY-VCH}

EDX detector from Oxford instruments was used when required. For the cross-sectional SEM images, a FEI QuantaFEG650 microscope was used. XRD patterns from $12^{\circ}$ to $60^{\circ}$ were measured using a Panalytical X'Pert MPD pro diffractometer at a scan rate of $2^{\circ} \min ^{-1}$. The insitu temperature measurements were recorded using a V-MOLE compact thermal profiler (at 2 $\mathrm{Hz}$ ) with the probe attached to the PeLED cathode using Kapton tape. Infrared thermography images were taken using a FLIR series E5 camera.

\section{Supporting Information}

Supporting Information is available from the Wiley Online Library or from the author.

\section{Acknowledgements}

The authors thank Mr. Pradeep Panditha for the help with temperature and photoluminescence measurements. We thank Mr. Ahmed Fawzy for preparing the encapsulation foils. Finally, thanks to Mr. Jack van Glabbeek for help with thermal evaporation. This work was financially supported by the European Commission through the Horizon 2020 Marie Sklodowska-Curie ITN-INFORM project (Grant Agreement No. 675867). HJB acknowledges the Spanish Ministry of Science, Innovation and Universities for support via the Unidad de Excelencia María de Maeztu MDM-2015-0538, and MAT2017-88821-R and the Generalitat Valenciana (Prometeo/2016/135).

Received: ((will be filled in by the editorial staff))

Revised: ((will be filled in by the editorial staff)) Published online: ((will be filled in by the editorial staff))

\section{References}

[1] H. Cho, S.-H. Jeong, M.-H. Park, Y.-H. Kim, C. Wolf, C.-L. Lee, J. H. Heo, A. Sadhanala, N. Myoung, S. Yoo, S. H. Im, R. H. Friend, T.-W. Lee, Science 2015, 350, 1222.

[2] X. Yu Chin, A. Perumal, A. Bruno, N. Yantara, S. A. Veldhuis, L. Martínez-Sarti, B. 


\section{WILEY-VCH}

Chandran, V. Chirvony, A. Shu-Zee Lo, J. So, C. Soci, M. Grätzel, H. J. Bolink, N.

Mathews, ad G. Subodh Mhaisalkar, Energy Environ. Sci 2018, 11, 1770.

[3] W. Xu, Q. H. Hu, S. Bai, C. Bao, Y. Miao, Z. Yuan, T. Borzda, A. J. Barker, E.

Tyukalova, Z. H. Hu, M. Kawecki, H. Wang, Z. Yan, X. Liu, X. S. Shi, K. Uvdal, M.

Fahlma, F. Gao, Nat. Photonics 2019, DOI: 10.1038/s41566- 019-0390-x.

[4] G. Xing, N. Mathews, S. S. Lim, N. Yantara, X. Liu, D. Sabba, M. Grätzel, S.

Mhaisalkar, T. C. Sum, Nat. Mater. 2014, 13, 476.

[5] Y. Jia, R. A. Kerner, A. J. Grede, B. P. Rand, N. C. Giebink, Nat. Photonics 2017, 11, 784.

[6] Y. H. Kim, H. Cho, J. H. Heo, T. S. Kim, N. S. Myoung, C. L. Lee, S. H. Im, T. W. Lee, Adv. Mater. 2015, 27, 1248.

[7] L. Protesescu, S. Yakunin, M. I. Bodnarchuk, F. Krieg, R. Caputo, C. H. Hendon, R. X. Yang, A. Walsh, M. V. Kovalenko, Nano Lett. 2015, 15, 3692.

[8] C. Wehrenfennig, G. E. Eperon, M. B. Johnston, H. J. Snaith, L. M. Herz, Adv. Mater. 2014, 26, 1584.

[9] Z. Li, Y. Zhao, X. Wang, Y. Sun, Z. Zhao, Y. Li, H. Zhou, Q. Chen, Joule 2018, 2, 1559.

[10] L. Meng, E. P. Yao, Z. Hong, H. Chen, P. Sun, Z. Yang, G. Li, Y. Yang, Adv. Mater. 2017, 29, 1.

[11] V. Prakasam, F. Di Giacomo, R. Abbel, D. Tordera, M. Sessolo, G. Gelinck, H. J. Bolink, ACS Appl. Mater. Interfaces 2018, 10, 41586.

[12] K. Lin, J. Xing, L. N. Quan, F. P. G. de Arquer, X. Gong, J. Lu, L. Xie, W. Zhao, D. Zhang, C. Yan, W. Li, X. Liu, Y. Lu, J. Kirman, E. H. Sargent, Q. Xiong, Z. Wei, Nature 2018, 562, 245.

[13] M. Yuan, L. N. Quan, R. Comin, G. Walters, R. Sabatini, O. Voznyy, S. Hoogland, Y. Zhao, E. M. Beauregard, P. Kanjanaboos, Z. Lu, D. H. Kim, E. H. Sargent, Nat. 


\section{WILEY-VCH}

Nanotechnol. 2016, 11, 1.

[14] M. Ban, Y. Zou, J. P. H. Rivett, Y. Yang, T. H. Thomas, Y. Tan, T. Song, X. Gao, D. Credington, F. Deschler, H. Sirringhaus, B. Sun, Nat. Commun. 2018, 9, 3892.

[15] L. Zhao, K. M. Lee, K. Roh, S. U. Z. Khan, B. P. Rand, Adv. Mater. 2019, 31, 1805836.

[16] Y. Cao, N. Wang, H. Tian, J. Guo, Y. Wei, H. Chen, Y. Miao, W. Zou, K. Pan, Y. He, H. Cao, Y. Ke, M. Xu, Y. Wang, M. Yang, K. Du, Z. Fu, D. Kong, D. Dai, Y. Jin, G. Li, H. Li, Q. Peng, J. Wang, W. Huang, Nature 2018, 562, 249.

[17] L. Zhao, J. Gao, Y. H. L. Lin, Y. W. Yeh, K. M. Lee, N. Yao, Y. L. Loo, B. P. Rand, Adv. Mater. 2017, 29, 1.

[18] S. Bae, S. Kim, S. W. Lee, K. J. Cho, S. Park, S. Lee, Y. Kang, H. S. Lee, D. Kim, J. Phys. Chem. Lett. 2016, 7, 3091.

[19] H. Kim, L. Zhao, J. S. Price, A. J. Grede, K. Roh, A. N. Brigeman, M. Lopez, B. P. Rand, N. C. Giebink, Nat. Commun. 2018, 9, 4893.

[20] J. M. Frost, K. T. Butler, F. Brivio, C. H. Hendon, M. van Schilfgaarde, A. Walsh, Nano Lett. 2014, 14, 2584.

[21] J. H. Noh, S. H. Im, J. H. Heo, T. N. Mandal, S. Il Seok, Nano Lett. 2013, 13, 1764.

[22] P. van de Weijer, P. C. P. Bouten, S. Unnikrishnan, H. B. Akkerman, J. J. Michels, T. M. B. van Mol, Org. Electron. physics, Mater. Appl. 2017, 44, 94.

[23] N. Droseros, G. Longo, J. C. Brauer, M. Sessolo, H. J. Bolink, N. Banerji, ACS Energy Lett. 2018, 3, 1458.

[24] M. R. Phillips, Microchim. Acta 2006, 155, 51.

[25] S. S. Mali, C. S. Shim, C. K. Hong, NPG Asia Mater. 2015, 7, e208.

[26] J. A. Christiansen, On the Reaction Between Hydrogen and Bromine, Pergamon Press Ltd, 2013.

[27] J. G. Aston, C. W. Siller, G. H. Messerly, J. Am. Chem. Soc. 1937, 59, 1743. 


\section{WILEY-VCH}

[28] W. F. Giauque, R. Wiebe, J. Am. Chem. Soc. 1928, 50, 2193.

[29] B. Brunetti, C. Cavallo, A. Ciccioli, G. Gigli, A. Latini, Sci. Rep. 2016, 6, 31896.

[30] L. A. Frolova, N. N. Dremova, P. A. Troshin, Chem. Commun. 2015, 51, 14917.

[31] H. Lee, D. Ko, C. Lee, ACS Appl. Mater. Interfaces 2019, 11, 11667.

[32] L. Zhao, R. A. Kerner, Z. Xiao, Y. L. Lin, K. M. Lee, J. Schwartz, B. P. Rand, ACS Energy Lett. 2016, 1, 595-602.

[33] H. Wang, X. Zhang, Q. Wu, F. Cao, D. Yang, Y. Shang, Z. Ning, L. Zhang, W. Zhang, W. Zheng, Y. Yan, Nat. Commun. 2019, 10, 1. 


\section{WILEY-VCH}

a)

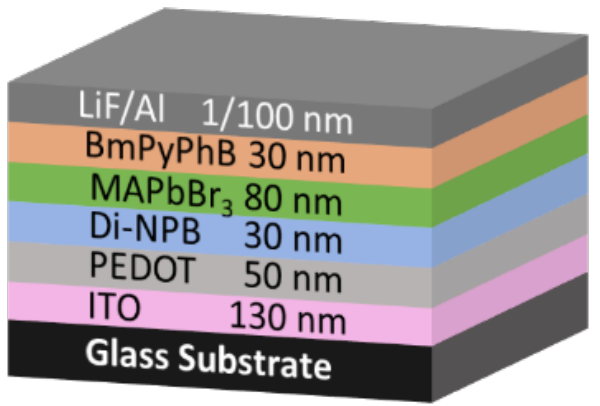

c)

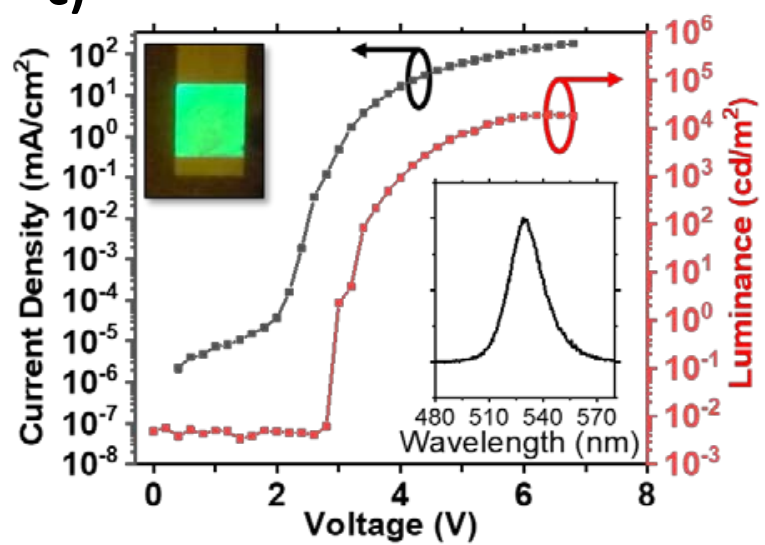

b)

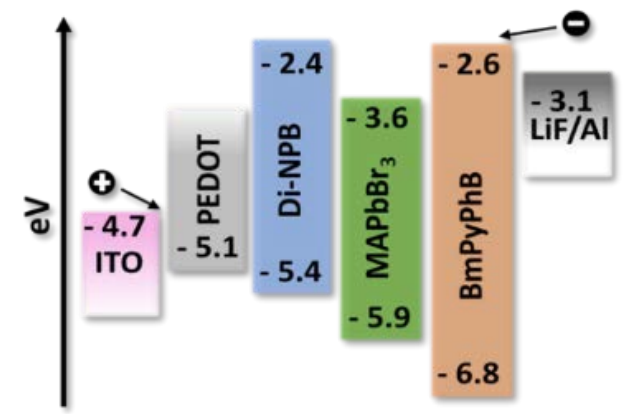

d)

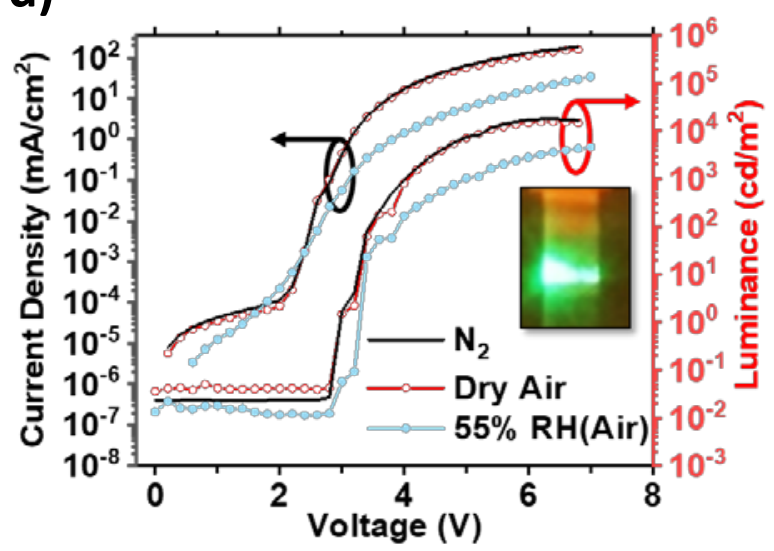

Figure 1. a) Schematic device architecture of the PeLED. b) Energy level diagram of the different layers of the PeLED. c) J-V-L curve of a typical device used in this study prepared under $\mathrm{N}_{2}$ environment. Insets: Photo of a working PeLED at $5 \mathrm{~V}$ bias and electroluminescence spectrum. d) J-V-L curves of the devices fabricated under $\mathrm{N}_{2}$ (black lines), dry air (red lines) and 55\% RH air (cyan lines) environment. Inset: Photo of a PeLED fabricated under humid air with $55 \% \mathrm{RH}$ at $5 \mathrm{~V}$ bias exhibiting non-uniform emission. The active area of the PeLEDs is $16 \mathrm{~mm}^{2}$ 
a)

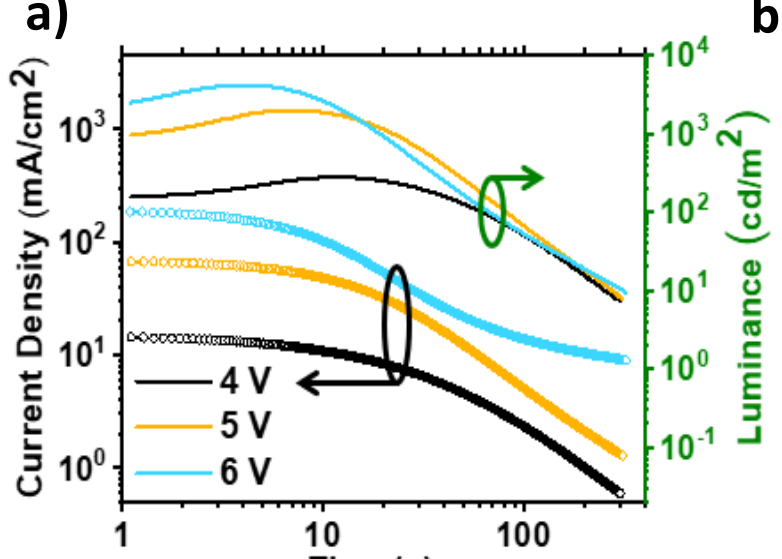

c)

Time (s)

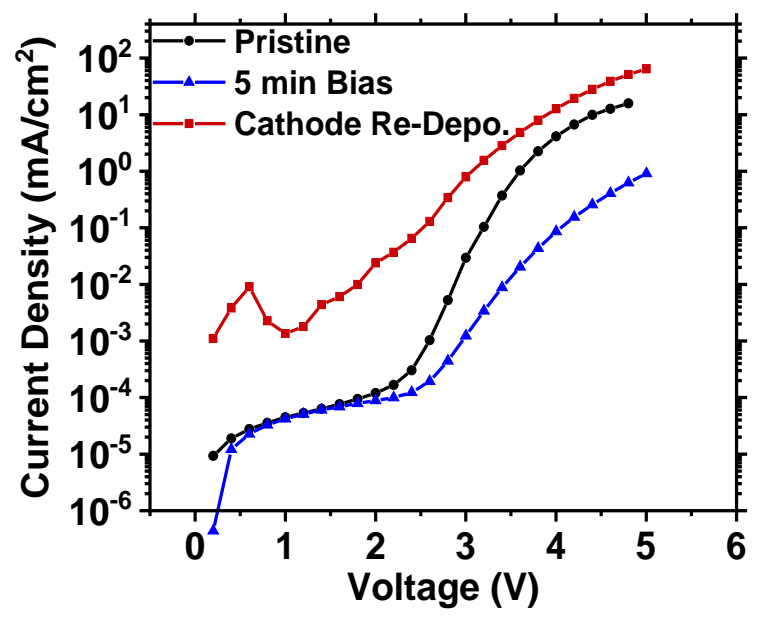

b) Bright Field Pristine

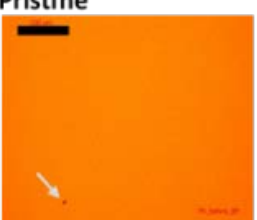

5 mins bias @ $5 \mathrm{~V}$
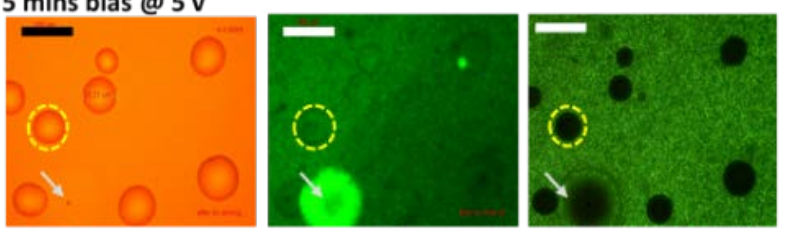

d)

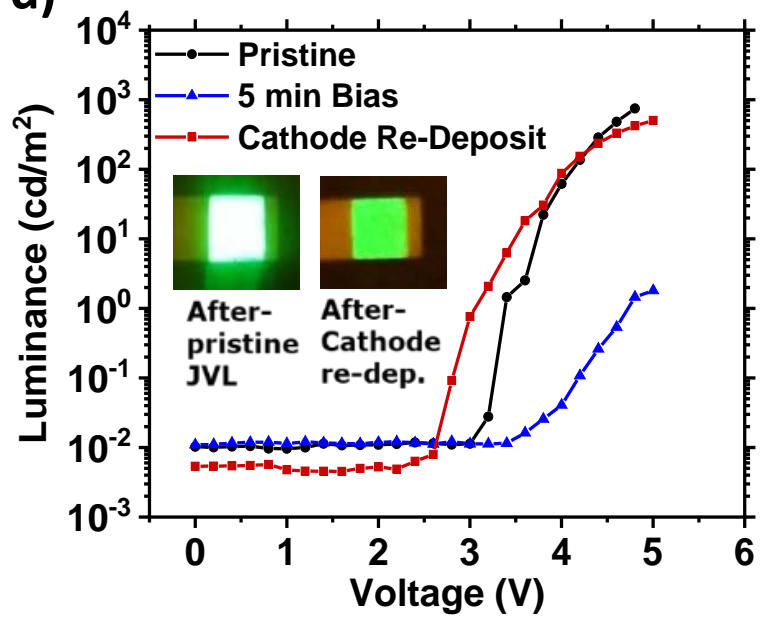

Figure 2. a) Current density (circles) and luminance (lines) vs. time of PeLEDs measured at various continuous bias voltages for 5 mins. b) Microscopic images of a PeLED in bright field, photoluminescence (excited at $405 \mathrm{~nm}$ ) and electroluminescence modes taken at $0 \mathrm{~s}$ (top) and after $5 \mathrm{~min}$ (bottom) of biasing at $5 \mathrm{~V}$. The scale bars represent $100 \mu \mathrm{m}$. The white arrows point at a particle for tracking. The yellow ring tracks one of the formed features after biasing. c) Current density-voltage; d) luminance-voltage of a PeLED freshly prepared (black line), after 5 min bias (5 V) (blue line) and after stripping and re-depositing the cathode (red line). Inset: Photo of the PeLED taken at $5 \mathrm{~V}$ after the first JVL sweep and after the cathode re-deposition. 


\section{WILEY-VCH}

a)

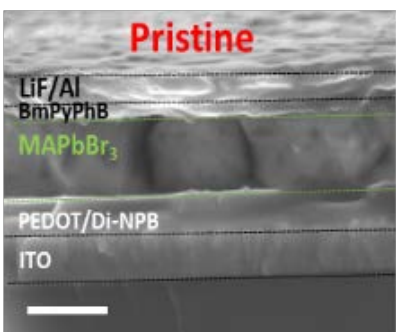

b)

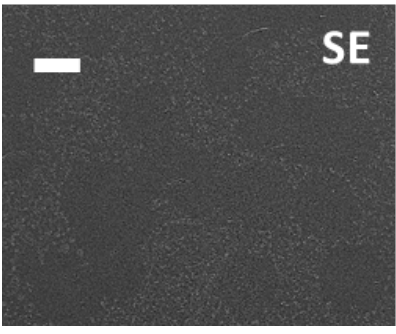

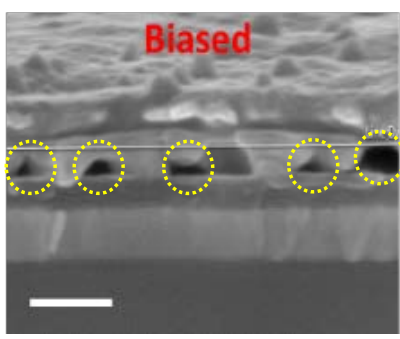

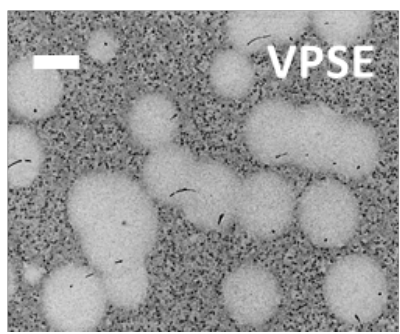

c)
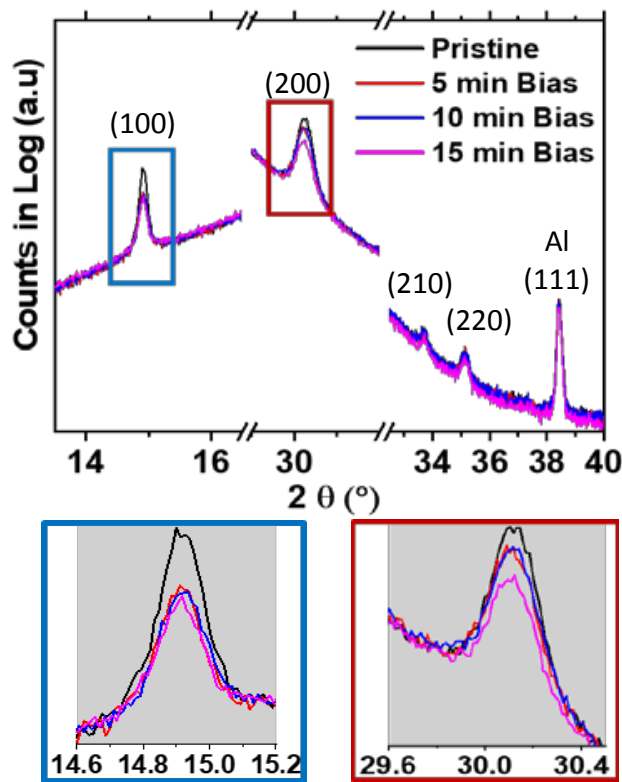

$\theta \theta\left(^{\circ}\right)$

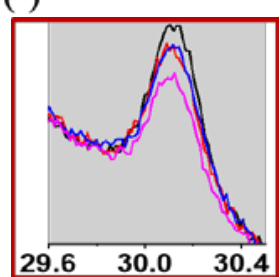

Figure 3. a) Cross-sectional SEM images of a PeLED before and after biasing. The cavities formed in biased device is highlighted with yellow circles. The scale bars represent $250 \mathrm{~nm}$. The EDX line data of the biased device is shown in Figure S8. b) SEM topographic images of a biased PeLED after peeling off the cathode taken using SE and VPSE detectors at the same spot. The scale bars represent $100 \mu \mathrm{m}$. c) XRD patterns of the encapsulated PeLED stack at pristine and biased (5 V) conditions for various time durations (5, 10 and $15 \mathrm{~min}$ ) with the crystallographic planes on top. The magnified portions of the XRD spectra are shown below the main image. 


\section{WILEY-VCH}

\section{a)}

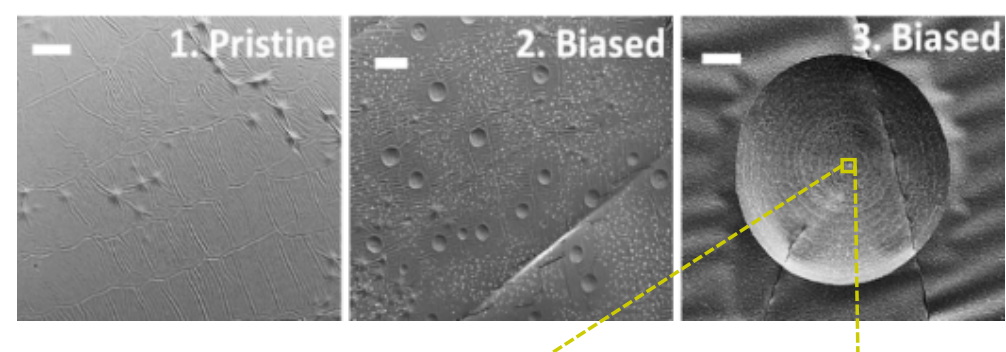

b)

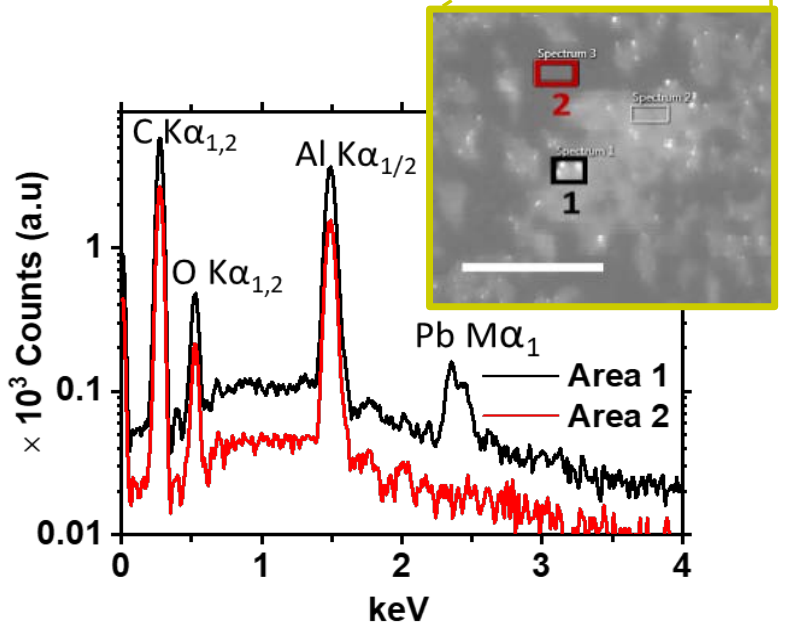

Figure 4. a) SEM images of the $\mathrm{Al}$ cathodes stripped off from a 1. pristine PeLED, 2. biased PeLED (scale bars - $200 \mu \mathrm{m}$ ) and 3. a biased PeLED at higher magnification (scale bar - 20 $\mu \mathrm{m})$. The wrinkles in the images are due to the peeling foil. b) EDX spectra of the areas enclosed by the black box (Area 1) and red box (Area 2) highlighted in the inset SEM image. The inset is a magnified portion (bound by the enclosed box) of one of the valleys in the stripped $\mathrm{Al}$ cathode. The scale bar represents $2.5 \mu \mathrm{m}$. 


\section{TOC Entry}

Degradation mechanisms in methylammonium lead bromide perovskite light emitting diode is systematically studied. The degradation is due to the decomposition of the perovskite layer under electric bias, leading to formation of gaseous methylamine and hydrogen bromide which locally delaminates the cathode from the device. Stripping and re-depositing a new cathode restores the current injection and luminance.

Keyword: Perovskite LEDs

Vittal Prakasam, Daniel Tordera, Henk J. Bolink, Gerwin Gelinck*

Degradation Mechanisms in Organic Lead Halide Perovskite Light-Emitting Diodes

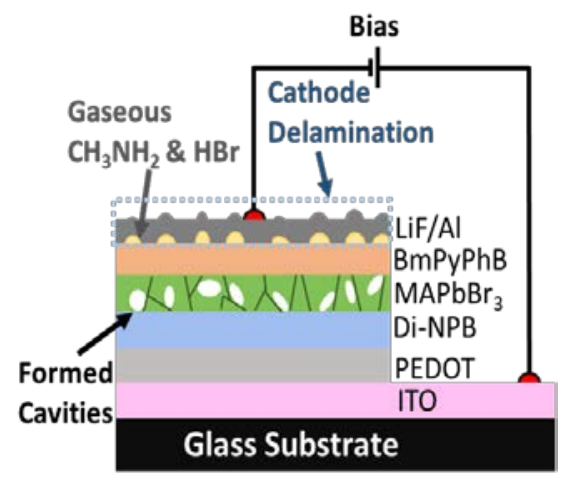

Copyright WILEY-VCH Verlag GmbH \& Co. KGaA, 69469 Weinheim, Germany, 2018. 